\title{
(2) OPEN ACCESS \\ Stage 4 pancreatic adenocarcinoma harbouring an FGFR2-TACC2 fusion mutation with complete response to erdafitinib a pan-fibroblastic growth factor receptor inhibitor
}

\author{
Donald Poon, ${ }^{1}$ Min Han Tan, ${ }^{2}$ Damian Khor $^{3}$
}

${ }^{1}$ Medical Oncology, Mount Elizabeth Novena Specialist Centre, Singapore

2Lucence Diagnostics, Singapore ${ }^{3}$ Radiology, Nuclear Medicine, Advanced Medical Imaging, Singapore

\section{Correspondence to} Dr Donald Poon; donald.poon@dyhpoon.com

Accepted 22 August 2021

\section{Check for updates}

(c) BMJ Publishing Group Limited 2021. Re-use permitted under CC BY-NC. No commercial re-use. See rights and permissions. Published by BMJ.

To cite: Poon D, Tan MH, Khor D. BMJ Case Rep

2021:14:e244271.

doi:10.1136/bcr-2021-

244271

\section{SUMMARY}

We report a case of a frail 68-year-old woman with stage 4 pancreatic carcinoma harbouring a fibroblastic growth factor receptor 2 (FGFR2) fusion who achieved a durable complete response after treatment with erdafitinib a pan-FGFR inhibitor. The FGFR2-TACC2 fusion was detected on comprehensive tumour somatic mutation profiling. There is ongoing complete response at 10 months after initiation of erdafitinib. Transient central serous retinopathy, grade 2 hyperphosphataemia and diarrhoea were the adverse events encountered.

\section{BACKGROUND}

Less than $20 \%$ of patients diagnosed with pancreatic cancer have operable disease and a majority of them have either locally advanced or metastatic disease with a median survival of less than 3-6 months. ${ }^{1}$ In a recent study comparing combination therapy leucovorin, 5-fluorouracil (5FU), oxaliplatin and irinotecan (FOLFIRINOX) versus gemcitabine for metastatic pancreatic cancer, ${ }^{2}$ the median overall survival was 11.1 months in the FOLFORINOX group compared with 6.8 months in the gemcitabine group (HR for death 0.57 ; 95\% CI 0.45 to $0.73 ; \mathrm{p}<0.001)$. In another study comparing the combination of gemcitabine and nanoparticle albumin-bound paclitaxel (nab-paclitaxel) versus single agent gemcitabine, ${ }^{3}$ it demonstrated a median survival of 8.5 months in the nabpaclitaxel and gemcitabine combination compared with 6.7 months in the gemcitabine monotherapy group (HR for death, $0.72 ; 95 \%$ CI 0.62 to 0.83 ; $\mathrm{p}<0.001)$. These combination chemotherapy regimens are usually used to treat patients with good performance status-Eastern Congress Oncology Grade (ECOG) 0-1.

In the group of patients with germline BRCAmutated metastatic pancreatic cancers, poly(adenosine diphosate-ribose) polymerase (PARP) inhibitor olaparib when given as maintenance therapy after first line platinum-based chemotherapy imparted longer progression-free survival (PFS) compared with placebo. ${ }^{5}$ Complete response in metastatic pancreatic cancer with germline BRCA mutation when treated with PARP inhibitor olaparib has been reported. ${ }^{6}$

In the National Comprehensive Cancer Network (NCCN) Practice Guidelines, gemcitabine monotherapy is recommended treatment for advanced pancreatic cancer in patients with poor performance status. ${ }^{7}$ S-1 monotherapy (median survival of 9.7 months) was shown to be non-inferior to gemcitabine alone (median survival of 8.8 months) with HR of $0.96 ; 95 \%$ CI 0.78 to 1.18 in a phase III study conducted in Japan and Taiwan. ${ }^{8}$ Complete response to monotherapy $\mathrm{S}-1$ in this context has also been reported. ${ }^{9}$

Tumour somatic gene profiling using nextgeneration sequencing is recommended for patients with locally advanced or metastatic pancreatic adenocarcinoma in the NCCN Practice Guidelines. $^{7}$ These guidelines stipulated the inclusion of specific fusions (ALK, NRG1, NTRK, ROS1), mutations (BRAF, BRCA1/2, HER2, KRAS, PALB2) and mismatch repair deficiency. NCCN Practice Guidelines further recommended peripheral blood cellfree DNA testing to be considered if tumour tissue testing is not feasible.

Our case report highlights the importance of screening for fibroblastic growth factor receptor (FGFR) fusion and mutation in pancreatic cancers as there are available effective treatment options in FGFR inhibitors. The prevalence of FGFR fusions and mutations in pancreatic cancer is estimated to be about $5 \% .^{10}$ If this favourable response to an FGFR inhibitor in pancreatic cancers with FGFR fusion as described in our case report is consistently replicated, it will significantly improve the outlook in patients with advanced pancreatic cancer harbouring such an FGFR fusion.

\section{CASE PRESENTATION}

Our patient who is a 68-year-old ethnic Chinese woman had a prior history of intraductal papillary mucinous neoplasm (IPMN) manifesting as an $8 \mathrm{~mm}$ cystic side branch communicating with the main duct in the body and $4 \mathrm{~mm}$ cystic lesion in the uncinate process of the pancreas. These were detected incidentally on CT urogram when she presented with a complicated urinary tract infection in 2013. She has no other significant medical history of note. The IPMN was documented to be stable based on MRI after 1 year and in subsequent biennial MRI scan of the pancreas from 2013 to 2018.

She started experiencing abdominal discomfort in mid-2020. MRI scan done a month after onset of abdominal pain revealed a mass in the head of pancreas measuring $2.5 \mathrm{~cm}$ by $2.1 \mathrm{~cm}$ encasing both the superior mesenteric artery and vein. It had also 
compressed on the common bile duct causing biliary drainage obstruction and the tumour had invaded the third part of the duodenum. The radiological features of multiple liver nodules in segments 6, 7 and 8 were consistent with metastatic disease. The diagnosis of adenocarcinoma of the pancreas was confirmed by ultrasound guided biopsy of the segment 6 liver metastasis.

She started treatment with chemotherapy combination capecitabine/oxaliplatin $(\mathrm{CAPOX})^{11}$ in view of her poor performance status. Her performance status was ECOG 2 at presentation. After two cycles of CAPOX, she deteriorated further with severe loss of appetite, abdominal pain and rapid loss of weight of $10 \mathrm{~kg}$ within 1 month. Progressive disease was then documented on CT imaging. The tumour specimen obtained from the biopsy of the liver metastasis was sent for comprehensive tumour genetic profiling. When she was admitted to hospital for intravenous hydration and sodium repletion, her performance status was ECOG 4. Her inability to retain sufficient enteral nutrition as a result of the progressive duodenal obstruction from tumour invasion resulted in grade 3 hyponatraemia (serum sodium $=120 \mathrm{mmol} / \mathrm{L}$ ).

Tumour profiling detected an FGFR2-TACC2 fusion in the tumour specimen and oral erdafitinib $5 \mathrm{mg}$ per day was started on knowledge of the profiling result. The specific FGFR2-TACC2 (NM_000141)-TACC2(NM_006997) fusion was at (F17; T7). We postulated that this fusion may be associated with a clinical response to FGFR inhibitors such as erdafitinib. There was complete resolution of nausea and abdominal pain within 3 days of starting erdafitinib. She was discharged well and walked out of hospital without assistance after 1 week of treatment using erdafitinib.

\section{INVESTIGATIONS}

CT scan evaluation which was done after the second cycle of CAPOX chemotherapy showed progressive disease. The primary pancreatic tumour encased both the superior mesenteric artery and vein, caused common bile duct obstruction and invaded the third part of the duodenum (figure 1). Multiple liver metastases were again detected (figure 2).

Tumour marker CA (carbohydrate antigen) 19-9 increased from baseline of $2139 \mathrm{U} / \mathrm{mL}$ (normal range $0-37 \mathrm{U} / \mathrm{mL}$ ) to $5516.3 \mathrm{U} / \mathrm{mL}$ after second cycle of CAPOX. Two weeks after initiation of erdafitinib, CA 19-9 dropped rapidly to $79.3 \mathrm{U} / \mathrm{mL}$ and then normalised with a value of $36.7 \mathrm{U} / \mathrm{mL}$ a month later.

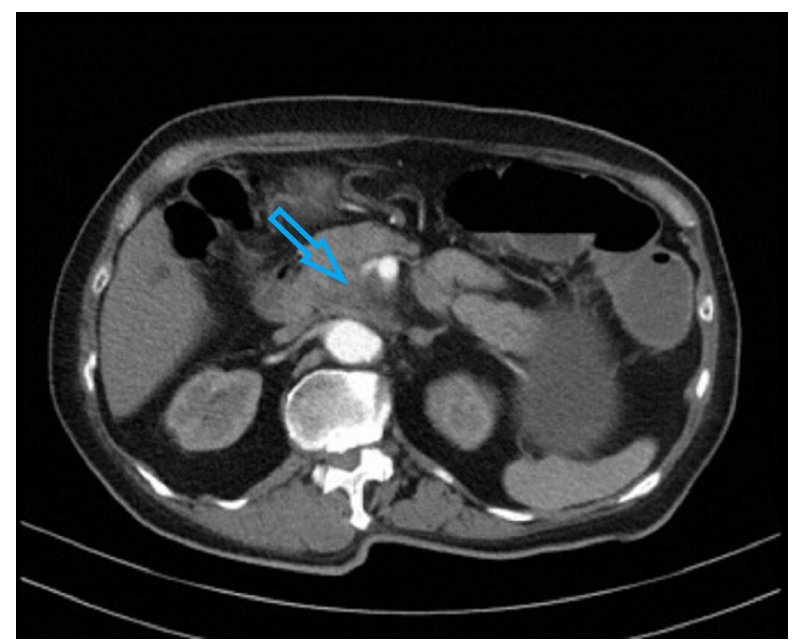

Figure 1 CT scan done at diagnosis showing encasement of superior mesenteric vessels indicated by arrow.

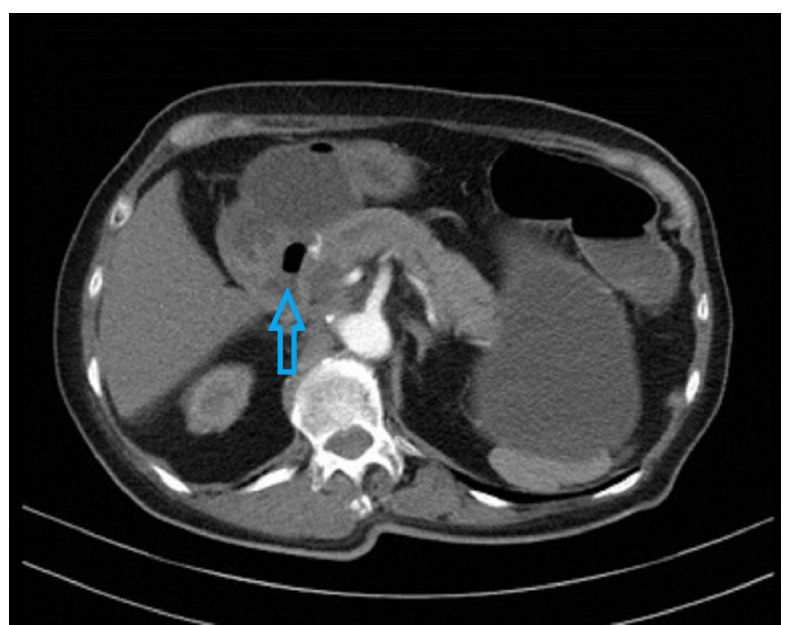

Figure 2 CT scan done at diagnosis showing tumour invading third part of duodenum indicated by arrow.

CA 19-9 continued to remain normal at 10 months after initiation of erdafitinib treatment.

Our patient's tumour FGFR2-TACC2 (NM_000141)-TACC2(NM_006997) fusion (F17; T7) was detected using FoundationOneCDx laboratory service from DNA extracted from the formalin-fixed, paraffin-embedded tumour biopsy specimen. FGFR 2 mutations are found in less than 1\% of pancreatic cancers. ${ }^{5}$ The tumour KRAS status was wild type, programmed cell death ligand 1 (PD-L1), tumour proportion score (TPS) and combined proportion score (CPS) score was 0 and microsatellite status was stable.

Repeat CT imaging 2 months after initiation of erdafitinib showed complete resolution of the primary pancreatic tumour and the liver metastases (figure 3). Corresponding positron emission tomography imaging demonstrated complete metabolic resolution in the head of pancreas (figure 4) and no other active metastatic disease elsewhere in the body (figure 5).

\section{TREATMENT}

Erdafitinib is a potent tyrosine kinase inhibitor of FGFR1-4. The drug has shown antitumour activity in preclinical models of different solid tumours, ${ }^{12}$ and in a phase 1 study involving patients with urothelial carcinoma and other tumour types with FGFR alterations. ${ }^{13}$ It is US Food Drug Administration approved

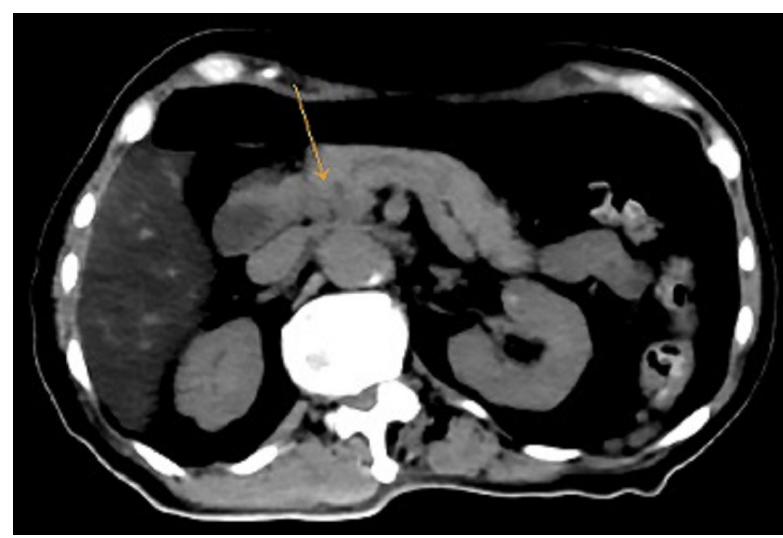

Figure 3 CT scan component of PET scan done after 2 months of erdafitinib treatment showing interval resolution of the previously seen neoplasm indicated by arrow in the pancreatic head. PET, positron emission tomography. 


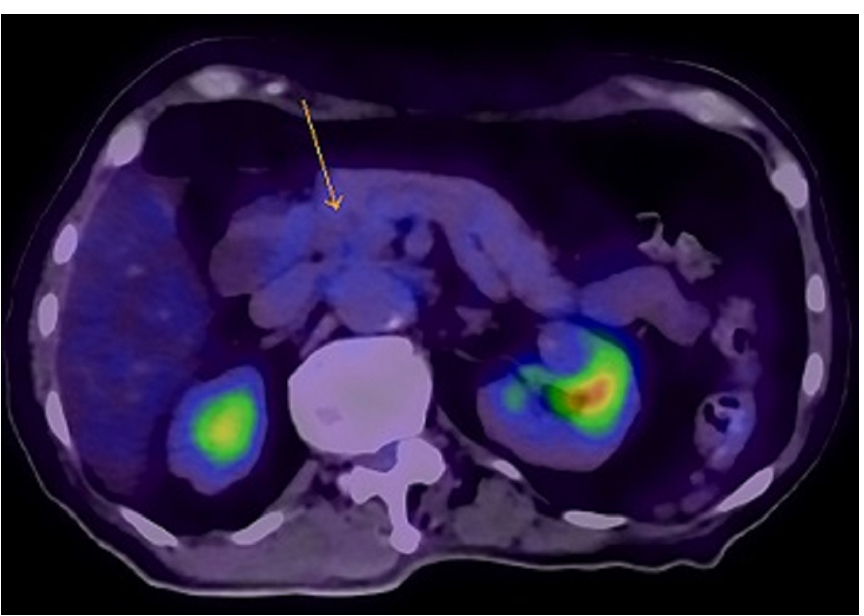

Figure 4 PET scan done after 2 months of erdafitinib treatment with no residual abnormal FDG uptake seen in primary tumour indicated by arrow. PET, positron emission tomography; FDG, fluorodeoxyglucose.

for the treatment of patients with advanced or metastatic urothelial carcinoma who have FGFR2 or FGFR3 alterations and have progressed after prior chemotherapy.

In view of the possibility of aggravation of hyponatraemia, nausea and vomiting, our patient was started on a reduced dose of erdafitinib (5 mg per day) compared with what is currently recommended for the initial treatment of advanced urothelial carcinoma (8 mg per day).

Serum phosphate level increased to $8.5 \mathrm{mg} / \mathrm{dL}$ (grade 2) 2 weeks after erdafitinib use and it reduced to less than $3 \mathrm{mg} / \mathrm{dL}$ after 1-week cessation of erdafitinib and reinstatement at a lower dose of $4 \mathrm{mg}$ per day thereafter. The serum phosphate level has remained stable between 1.5 and $2 \mathrm{mg} / \mathrm{dL}$ at this dose level till time of writing with dietary phosphate restriction to less than $800 \mathrm{mg}$ of phosphate per day.

The grade 2 diarrhoea encountered at $5 \mathrm{mg}$ of erdafitinib per day resolved with loperamide per oral $4 \mathrm{mg} 3-4$ times per day and diarrhoea is no longer encountered with the current dose level of $4 \mathrm{mg}$ per day.

Transient central serous retinopathy was diagnosed when our patient had mild visual impairment 1 week after use of erdafitinib. It resolved when the erdafitinib dose level was reduced from $5 \mathrm{mg}$ to $4 \mathrm{mg}$ per day.

\section{OUTCOME AND FOLLOW-UP}

Our patient has returned to work and is presently free of symptoms. Performance status is ECOG 0 at present and she has regained her original weight prior to diagnosis of pancreatic carcinoma. She continues to be on erdafitinib at $4 \mathrm{mg}$ per day. Response evaluation CT scan will be performed every 3-4 months.

\section{DISCUSSION}

Bailey et al reported the findings of an integrated genomic analysis of 456 pancreatic ductal adenocarcinoma tumours and identified four subtypes of pancreatic adenocarcinoma (squamous, pancreatic progenitor, immunogenic and aberrantly differentiated endocrine exocrine). These four subtypes, though distinct in histopathological features, were associated with better survival prognosis in KRAS wild type tumours. ${ }^{14}$ Approximately $8 \%-10 \%$ of pancreatic ductal adenocarcinoma are KRAS wild type. Of clinical therapeutic relevance, targetable fusions were
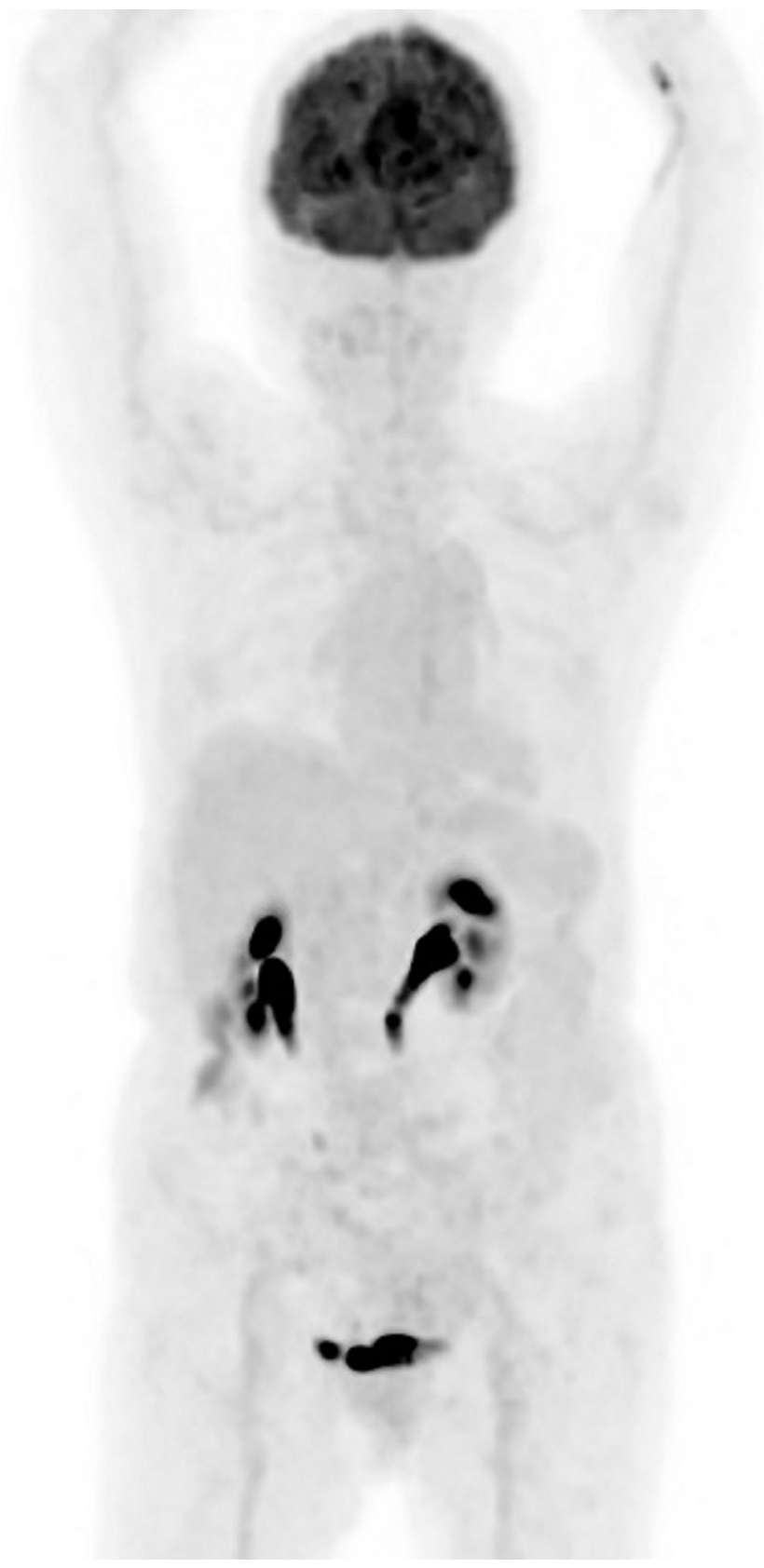

Figure 5 PET scan done after 2 months of erdafitinib treatment demonstrated no FDG-avid metastatic disease identified in body. PET, positron emission tomography; FDG, fluorodeoxyglucose.

found more often in KRAS wild type pancreatic adenocarcinoma tumours compared with KRAS mutated tumours. ${ }^{15}$ Treatment using the appropriate tyrosine kinase inhibitors targeting the relevant fusions and mutations found resulted in significant clinical responses. ${ }^{16}$

FGFR2 encodes a tyrosine kinase cell surface receptor which plays an important role in cell differentiation, growth and angiogenesis. ${ }^{17}$ Specifically FGFR2 alterations have been found in less than $1 \%$ of pancreatic cancers. ${ }^{17}$ The FGFR2TACC2 fusion (F17; T7) detected in our patient's tumour tissue is structurally similar to other characterised FGFR2 fusions including FGFR2-BICC1, FGFR2-CCDC6, FGFR2-AHCYL1, FGFR2-FRAG1, FGFR2-NOL4 and FGFR2-TACC3. These fusions have been shown to be activating oncogenes and are sensitive to FGFR inhibitors such as erdafitinib. Furthermore 
FGFR2 variants with alterations resulting in lack of the cytoplasmic domain encoded by exon 18 have been reported to be oncogenic in vitro, ${ }^{18}$ and therefore, the detected FGFR2TACC2 fusion (F17; T7) in our patient's tumour is an activating rearrangement.

Erdafitinib is a pan-FGFR1-4 inhibitor. In cholangiocarcinoma, good response is found in those tumours harbouring FGFR2 fusions, while FGFR2 mutations and amplifications seem to confer less sensitivity to erdafitinib. This was demonstrated in a phase 2 a study of erdafitinib in previously treated patients with FGFR-altered advanced cholangiocarcinoma. ${ }^{19}$ For those with FGFR2 or FGFR3 fusion positive cholangiocarcinoma, the median PFS was 12.7 months compared with the overall PFS of 5.6 months for the entire study cohort. A phase 1 study for erdafinitib in solid tumours reported a better response in tumours carrying FGFR mutations or gene fusions compared with the overall cohort, with an overall response rate (ORR) of 21\% (19/92) versus 11\% (21/187), respectively. ${ }^{20}$ The most responsive tumour types in this study were urothelial carcinoma and cholangiocarcinoma, with an ORR of 46\% (12/26) and 27\% (3/11), respectively, in response-evaluable patients with FGFR mutations or fusions. Of patients with FGFR altered cholangiocarcinoma treated with erdafitinib in another phase 1 study, including one patient with FGFR2 mutation and two patients with FGFR2 fusions, 27\% (3/11) exhibited PRs; the median duration of response was 11.4 months, and an additional 27\% (3/11) patients exhibited stable disease. ${ }^{21}$ The differential response in cholangiocarcinoma between FGFR fusions and other mutations was not seen in the phase 2 study of erdafitinib in urothelial carcinoma ${ }^{22}$; the response rate among the 74 patients with FGFR mutations in the selected-regimen group was $49 \%$, while among the 25 patients with FGFR fusions, the response rate was $16 \%$. In this study, less than half $(46 \%)$ of the patients reported having an adverse event of grade 3 or higher that was related to erdafitinib. Common events of grade 3 or higher including hyponatraemia (11\%), stomatitis $(10 \%)$, asthenia $(7 \%)$, central serous retinopathy $(3 \%)$ and hyperphosphataemia (2\%) were reported at the recommended daily dose of $8 \mathrm{mg}$ erdafitinib in the treatment of urothelial cancers. Our patient did not experience any grade 3 or higher adverse event at $5 \mathrm{mg}$ per day. The central serous retinopathy, grade 2 hyperphosphataemia and diarrhoea resolved with dose reduction to $4 \mathrm{mg}$ of erdafitinib per day.

Pancreatic adenocarcinoma tumours carrying FGFR2 fusions and mutations are not well represented in the published studies. More studies focusing on the efficacy of FGFR inhibitors such as erdafitinib in the treatment of pancreatic cancers with FGFR fusions and mutations especially in those tumours with KRAS wild type status should be done.

\section{Patient's perspective}

- Our patient's comments: 'I am grateful for this new lease of life after being diagnosed with stage 4 pancreatic cancer and especially what was originally informed to me as a diagnosis with a dismal prognosis of less than 1 year median survival, I am however concerned about the cost of treatment after the drug early access programme ends as I do not have comprehensive health insurance coverage.'

- The sponsor has kindly committed to continuing with the access programme in provision of erdafitinib until disease progression for our patient.
Learning points

- As corroborated by established data in other tumour types such as cholangiocarcinoma and urothelial carcinoma, advanced pancreatic cancer harbouring fibroblastic growth factor receptor 2 (FGFR2) altering mutations and fusions may benefit from use of FGFR inhibitors such as erdafitinib.

- Profiling of pancreatic adenocarcinoma especially in those with KRAS wild-type status is important to look for actionable mutations and fusions which may be effectively treated using available targeted therapeutic agents especially if the patient is frail and unfit for systemic chemotherapy.

- Hyperphosphataemia and central serous retinopathy are potentially serious side effects of erdafitinib with a reported incidence of $2 \%$ and $3 \%$, respectively, of grade 3 or higher of such adverse events at a daily dose of $8 \mathrm{mg}$ erdafitinib. The hyperphosphataemia and central serous retinopathy resolved with dose reduction to $4 \mathrm{mg}$ per day of erdafitinib without interruption in its administration.

Acknowledgements The authors thank Janssen Pharmaceutical for allowing use of erdafitinib under a named-patient compassionate early access programme in Singapore.

Contributors Planning, conception/design of the work: DP. Acquisition, analysis and interpretation of data: DP, MHT and DK. Drafting the work and revising it critically for important intellectual content: DP, MHT and DK. Final approval of the version published and agree to be accountable for all aspects of the work in ensuring that questions related to the accuracy or integrity of any part of the work are appropriately investigated and resolved: DP, MHT and DK.

Funding The authors have not declared a specific grant for this research from any funding agency in the public, commercial or not-for-profit sectors.

Competing interests None declared.

Patient consent for publication Obtained.

Provenance and peer review Not commissioned; externally peer reviewed.

Open access This is an open access article distributed in accordance with the Creative Commons Attribution Non Commercial (CC BY-NC 4.0) license, which permits others to distribute, remix, adapt, build upon this work non-commercially, and license their derivative works on different terms, provided the original work is properly cited and the use is non-commercial. See: http://creativecommons.org/ licenses/by-nc/4.0/

\section{REFERENCES}

1 Siegel RL, Miller KD, Jemal A. Cancer statistics, 2018. CA Cancer J Clin 2018;68:7-30.

2 Conroy T, Desseigne F, Ychou M, et al. Folfirinox versus gemcitabine for metastatic pancreatic cancer. N Eng/ J Med 2011;364:1817-25.

3 Von Hoff DD, Ervin T, Arena FP, et al. Increased survival in pancreatic cancer with nabpaclitaxel plus gemcitabine. N Eng/ J Med 2013;369:1691-703.

4 Conill C, Verger E, Salamero M. Performance status assessment in cancer patients. Cancer 1990;65:1864-6.

5 Golan T, Hammel P, Reni M, et al. Maintenance olaparib for germline BRCA-mutated metastatic pancreatic cancer. N Engl J Med 2019;381:317-27.

6 Wang H, Mao C, Li N, et al. A case report of a dramatic response to olaparib in a patient with metastatic pancreatic cancer harboring a germline BRCA2 mutation. Medicine 2019;98:e17443.

7 Tempero MA, Malafa MP, Al-Hawary M. NCCN clinical practice guidelines in oncology: pancreatic adenocarcinoma. version 2, 2021. Available: nccn.org [Accessed 27 Jun 2021].

8 Ueno H, loka T, Ikeda M, et al. Randomized phase III study of gemcitabine plus S-1, S-1 alone, or gemcitabine alone in patients with locally advanced and metastatic pancreatic cancer in Japan and Taiwan: GEST study. J Clin Oncol 2013:31:1640-8.

9 Saso K, Murata M, Asai K, et al. [A case of pancreatic cancer with multiple liver metastases that developed postoperatively and showed a complete response with S-1 monotherapy]. Gan To Kagaku Ryoho 2015;42:1620-2.

10 Helsten T, Elkin S, Arthur E, et al. The FGFR landscape in cancer: analysis of 4,853 tumors by next-generation sequencing. Clin Cancer Res 2016;22:259-67.

11 Bullock A, Stuart K, Jacobus S, et al. Capecitabine and oxaliplatin as first and second line treatment for locally advanced and metastatic pancreatic ductal adenocarcinoma. J Gastrointest Oncol 2017;8:945-52. 
12 Perera TPS, Jovcheva E, Mevellec L, et al. Discovery and pharmacological characterization of JNJ-42756493 (erdafitinib), a functionally selective small-molecule FGFR family inhibitor. Mol Cancer Ther 2017;16:1010-20.

13 Tabernero J, Bahleda R, Dienstmann R, et al. Phase I dose-escalation study of JNJ42756493, an oral pan-fibroblast growth factor receptor inhibitor, in patients with advanced solid tumors. J Clin Oncol 2015;33:3401-8.

14 Bailey P, Chang DK, Nones K, et al. Genomic analyses identify molecular subtypes of pancreatic cancer. Nature 2016;531:47-52.

15 Fusco MJ, Saeed-Vafa D, Carballido EM, et al. Identification of targetable gene fusions and structural rearrangements to foster precision medicine in KRAS wild-type pancreatic cancer. JCO Precis Oncol 2021;5:65-74.

16 Jones MR, Williamson LM, Topham JT, et al. NRG1 gene fusions are recurrent, clinically actionable gene rearrangements in KRAS-wild type pancreatic ductal adenocarcinoma. Clin Cancer Res 2019;25:clincanres.0191.2019.
17 Powers CJ, McLeskey SW, Wellstein A. Fibroblast growth factors, their receptors and signaling. Endocr Relat Cancer 2000;7:165-97.

18 Wu Y-M, Su F, Kalyana-Sundaram S, et al. Identification of targetable FGFR gene fusions in diverse cancers. Cancer Discov 2013:3:636-47.

19 Park JO, Feng Y-H, Chen Y-Y, et al. Updated results of a phase IIA study to evaluate the clinical efficacy and safety of erdafitinib in Asian advanced cholangiocarcinoma (CCA) patients with FGFR alterations. JCO 2019;37:4117.

20 Bahleda R, Italiano A, Hierro C, et al. Multicenter phase I study of erdafitinib (jnj42756493), oral pan-fibroblast growth factor receptor inhibitor, in patients with advanced or refractory solid tumors. Clin Cancer Res 2019;25:4888-97.

21 Soria J-C, Strickler JH, Govindan R, et al. Safety and activity of the pan-fibroblast growth factor receptor (FGFR) inhibitor erdafitinib in phase 1 study patients (PTS) with molecularly selected advanced cholangiocarcinoma (CCA). JCO 2017;35:4074

22 Loriot $\mathrm{Y}, \mathrm{Necchi} A$, Park SH, et al. Erdafitinib in locally advanced or metastatic urothelial carcinoma. N Engl J Med 2019;381:338-48.

Copyright 2021 BMJ Publishing Group. All rights reserved. For permission to reuse any of this content visit

https://www.bmj.com/company/products-services/rights-and-licensing/permissions/

BMJ Case Report Fellows may re-use this article for personal use and teaching without any further permission.

Become a Fellow of BMJ Case Reports today and you can:

Submit as many cases as you like

- Enjoy fast sympathetic peer review and rapid publication of accepted articles

Access all the published articles

Re-use any of the published material for personal use and teaching without further permission

Customer Service

If you have any further queries about your subscription, please contact our customer services team on +44 (0) 2071111105 or via email at support@bmj.com.

Visit casereports.bmj.com for more articles like this and to become a Fellow 